\title{
Roundness error measurement using teaching learning based optimization algorithm and com- parison with particle swarm optimization algorithm
}

\author{
M.R. Pratheesh Kumar ${ }^{a^{*}}$, P. Prasanna Kumaar ${ }^{\text {b }}$, R. Kameshwaranath ${ }^{\text {, }}$, R.Thasarathan ${ }^{\text {b }}$
}

${ }^{a}$ Assistant Professor, Department of Production Engineering, PSG College of Technology, Coimbatore-641004, India ${ }^{b}$ Under Graduate Student, Department of Production Engineering, PSG College of Technology, Coimbatore-641004, India C H R O N I C L E

Article history:

Received: May 1, 2018

Received in revised format: June

16, 2018

Accepted: August 27, 2018

Available online:

August 27, 2018

Keywords:

Roundness error

Teaching Learning Based Optimi-

zation

Particle Swarm Optimization

Minimum zone circle

Least square circle

\section{Introduction}

According to Lei et al. (2011) roundness error is the most important form error in cylindrical features which is the variation between measured circular profile and an ideal reference circle. Roundness error is defined as the minimum radial separation between two concentric circles which contain all the coordinate data measured over the manufactured part. There are four geometry measurement techniques which form the basic criteria to estimate the reference circle. They are (i) minimum circumscribed circle (MCC), (ii) maximum inscribed circle (MIC), (iii) minimum zone circle (MZC) and (iv) least square circle (LSC) method. The use of specific measurement method depends on whether it is inside diameter or outside diameter (Muralikrishnan \& Raja, 2009). Most of the precision assemblies use cylindrical features with close dimensional and form tolerances. The errors in cylindrical component are due to

* Corresponding author. Tel.: +91-9786417582

E-mail address: mrpratheesh@gmail.com (M.R.Pratheesh Kumar)

(C) 2018 by the authors; licensee Growing Science, Canada. doi: $10.5267 /$ j.ijdns.2018.8.003 
various uncertain factors in manufacturing processes (Farooqui et al., 2009). Roundness and cylindricity errors are to be effectively and efficiently evaluated as they are important for proper assembly of the component with its mating part and for functional requirements (Mekid \& Vacharanukul, 2011). Estimation of form error involves fitting of large number of data points based on some criteria which is time consuming and difficult to do manually. This leads to the need for efficient algorithms in order to simplify and accelerate the computation of form errors (Muralikrishnan \& Raja, 2009; Mekid \& Vacharanukul, 2011).

Du et al. (2014) applied particle swarm optimization algorithm to evaluate roundness error based on MZC method using the data points given by Wen et al. (1999). The performance of PSO algorithm was analyzed using inertia weight and two learning factors. The results were compared with the MZC results of a geometry-based method, Genetic Algorithm, the novel PSO with linearly varying inertia weight, and the LSC method using PSO. The algorithm was tested for different sample size and found that roundness error increases with an increase in sample size. Jywe et al. (1999) proposed a min - max problem for evaluating the form error of a circular profile. This algorithm provided the best results by solving simultaneous linear algebraic equations. Sun (2009) applied PSO algorithm for roundness measurement for the data points obtained using machine vision system. The experimental results showed that the PSO based method effectively solved the MIC, MZC and MCC problems and outperforms genetic algorithm (GA) based method in both accuracy and efficiency. Wen et al. (2006) proposed an effective GA for evaluating roundness error based on MZC, MIC, MCC and the LSC. The results of the algorithm proved its capability to provide optimal solution and found flexible such that it can be used for evaluating other type of form errors like straightness, flatness and cylindricity. Dhanish (2002) proposed a simple algorithm for evaluating roundness error based on MZC method using simple algebraic equations. The coordinate data obtained from a co-ordinate measuring machine (CMM) were transformed to linearize the equation and a program was developed using $\mathrm{C}++$. The results were compared with other published data sets and found to be correct. He et al. (2015) proposed a mathematical model based on distance function by considering second order terms for the evaluation of error in free - form surfaces by a combination of differential evolution algorithm and Nelder Mead (NM) algorithm. The results obtained from differential distance function and differential evolution algorithm were compared with LSC method and the new method was found to have better performance.

Non-traditional optimization algorithms such as GA, PSO, Ant Colony Optimization (ACO) and Simulated Annealing (SA) require proper tuning of algorithm specific parameters such as cross over, mutation probability, inertia weight, velocity, pheromone concentration, temperature and energy when used for form measurements. Tuning of these algorithm specific parameters is a critical factor in obtaining optimal results. Improper tuning of the algorithm-specific parameters will either increase the computational effort or yield a local optimal solution. In addition to tuning of the algorithm-specific parameters, the common control parameters also need to be tuned which further complicates the process. Rao and Patel (2013) developed Teaching Learning Based Optimization Algorithm (TLBO) to solve unconstrained optimization problems using control parameters like population size and number of generations. It does not require algorithm specific parameters to solve such problems. The results of the algorithm were compared with that of other heuristic algorithms and proved to be capable of providing better results than them. The authors proposed the concept of elitism in TLBO to further improve its performance.

The aim of this paper is to use TLBO, a population-based algorithm for roundness error measurement which does not require algorithm specific parameters and to compare its results in terms of number of iterations required to converge, computation time and the ability to provide minimum roundness error with that of other algorithms which require algorithm specific parameters. As this algorithm is not yet been tried to evaluate roundness error, it is attempted in this paper. The data points given by Jywe et al. (1999) were used for this purpose and the results of TLBO algorithm were compared with the results of Du et al. (2014). 


\section{Teaching Learning Based Optimization Algorithm (TLBO)}

TLBO is a teaching-learning process inspired algorithm developed by Rao \& Patel (2015) based on the influence of a teacher on the results of learners in a class. The algorithm follows two basic modes of the learning: (i) through the teacher (known as teacher phase) and (ii) sharing knowledge with the other learners (known as learner phase). In this optimization algorithm a group of learners is assumed as population ' $n$ ', different subjects learnt by the learners are considered as unique design variables ' $m$ ', a learner's result as the fitness value of the optimization problem and $\mathrm{M}$ as the mean results of the learners in a particular subject. The best solution in the entire population is known as the teacher. For the evaluation of roundness error, ten randomly generated LSC centers $(a, b)$ were considered as the population. The center coordinates $(a, b)$ is considered as design variables, $M$ is the mean of ten design variables $(a, b)$ and the minimum roundness error in the entire population is considered as the best solution. The implementation flowchart of TLBO algorithm given by Rao (2015) is suitably modified to evaluate roundness error and is given in Fig. 1.

\subsection{Teacher Phase}

In this phase, the mean result of the class in the subjects taught by the teacher is upgraded in the successive iteration to find the best value. The best result $P_{\text {bess }}$ in any iteration ' $i$ ' among all the subjects in the entire population, is considered as the result of the best learner. However, the teacher is considered as a highly qualified person, the best learner identified by the algorithm in any iteration is considered as the teacher in the next iteration. The difference between the result of the teacher for each subject and existing mean result of each subject is given by difference mean (1). Considering a learner $\mathrm{P}\left(\mathrm{a}_{1}, \mathrm{~b}_{1}\right)$

$$
\text { Difference Mean }=r\left(P_{\text {best }}-T_{F} M\right) \text {, }
$$

where, $T_{F}$ is the teaching factor which decides the value of mean to be changed which is specified as 1 and $r$ is the random number in the range $[0,1] . \mathrm{T}_{\mathrm{F}}$ is decided randomly with equal probability as,

$$
\mathrm{T}_{\mathrm{F}}=\operatorname{round}[1+\operatorname{rand}(0,1)\{2-1\}] \text {. }
$$

$\mathrm{T}_{\mathrm{F}}$ is not an algorithm specific parameter. It is generated randomly by the algorithm using teaching factor (2). It is found that the algorithm provides better result (Rao \& Patel, 2013) for the $T_{F}$ values 1 and 2. The existing solution is updated in teacher phase using difference mean according to (3).

$$
\mathrm{P}^{\prime}=\mathrm{P}+\text { Difference Mean, }
$$

where, $\mathrm{P}^{\prime}$ is the updated value of $\mathrm{P}$. Accept $\mathrm{P}^{\prime}$ if it gives better roundness value. All the accepted roundness values at the end of the teacher phase become the input to the learner phase.

\subsection{Learner Phase}

Learners improve their knowledge by interacting among themselves. A learner interacts with every other learner and tries to gain knowledge from them if they have more knowledge than him or her.

Considering the ten LSC centers obtained at the end of teacher phase as the learners which is the population size, the learner phase is expressed as updated value of the center $\left(a_{1}, b_{1}\right)$ in learner's phase (4) and (5). Select two centers $P\left(a_{1}, b_{1}\right)$ and $Q\left(a_{2}, b_{2}\right)$ randomly from the ten LSC centers such that $P \neq Q$. Since evaluation of roundness error is a minimization problem, if the roundness value obtained for the centre $\mathrm{P}$ is less than centre $\mathrm{Q}$ the algorithm updates the value of centre $\mathrm{P}$ using (4) otherwise the centre $\mathrm{P}$ is updated using (5).

$$
\begin{aligned}
& \mathrm{P}^{\prime \prime}=\mathrm{P}^{\prime}+\mathrm{r}\left(\mathrm{P}^{\prime}-\mathrm{Q}^{\prime}\right) \text {, if } \mathrm{F}\left(\mathrm{P}^{\prime}\right)<\mathrm{F}\left(\mathrm{Q}^{\prime}\right), \\
& \mathrm{P}^{\prime \prime}=\mathrm{P}^{\prime}+\mathrm{r}\left(\mathrm{Q}^{\prime}-\mathrm{P}^{\prime}\right) \text {, if } \mathrm{F}\left(\mathrm{Q}^{\prime}\right)<\mathrm{F}\left(\mathrm{P}^{\prime}\right),
\end{aligned}
$$

where, $\mathrm{P}^{\prime \prime}$ is the updated value of the center $\left(\mathrm{a}_{1}, \mathrm{~b}_{1}\right)$ in learner's phase. Accept $\mathrm{P}$ " if it gives a better function value. 


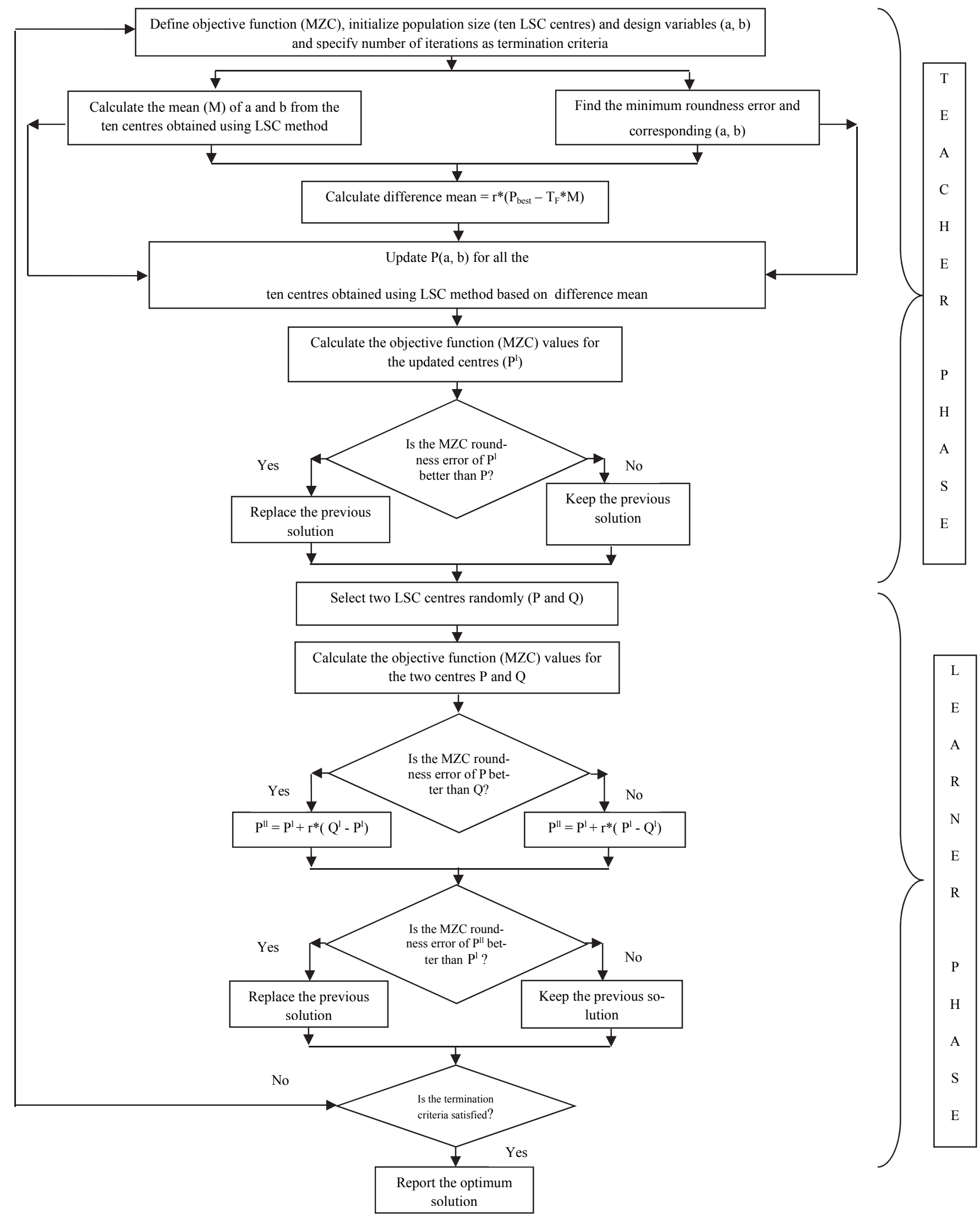

Fig. 1. Implementation flowchart of TLBO algorithm 


\section{Minimum zone circle method}

For a given set of data points, if all data points are on or between two concentric circles, the minimum radial separation between these two concentric circles is called the minimum zone solution. MZC method provides more accurate fitting results because it yields smaller zone value than other criteria and it is more consistent with standard definition of physical fitting (Wen et al., 2006). Fig. 2 shows the minimum zone circle fitted for a roundness profile.

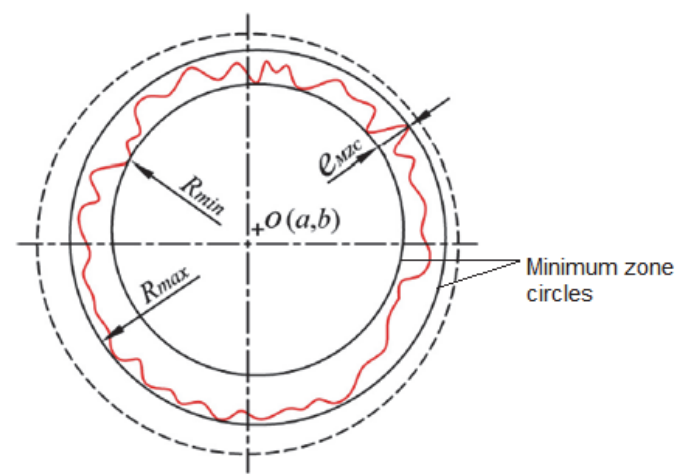

Fig. 2. Minimum zone circles fitted for roundness error (Du et al., 2014)

Since the roundness error value being evaluated should be minimum, it becomes a minimization problem. Assuming the center of MZC as $(a, b)$, the radial distance $r_{i}$ given in Eq. (6) from the data point $P_{i}\left(x_{i}, y_{i}\right)$ to the center is given by

$$
r_{i}=\sqrt{\left(x_{i}-a\right)^{2}+\left(y_{i}-b\right)^{2}} \text {. }
$$

The objective function (7) of MZC method is defined by

$$
\operatorname{Min}\left(E_{M Z C}\right)=\operatorname{Min}\left(R_{\max }-R_{\min }\right) \text {, }
$$

where, Min $\left(\mathrm{E}_{\mathrm{MZC}}\right)$ is the roundness error to be minimized according to $\mathrm{MZC}$ method, $\mathrm{R}_{\max }$ is the radius of the circumscribed circle forming the zone, $R_{\min }$ is the radius of the inscribed circle forming the zone. The objective function (7) is to be solved to find the center $(a, b)$ of the minimum zone circles such that the radial separation between them is minimum.

\section{Implementation of TLBO algorithm}

The initial population $(a, b)$ is obtained using LSC method. The centre coordinates $(a, b)$ are calculated using X coordinate of the LSC center (8) and Y coordinate of the LSC center (9).

$$
\begin{aligned}
& a=\frac{2}{n} \times \sum_{i=1}^{n} x \\
& b=\frac{2}{n} \times \sum_{i=1}^{n} y
\end{aligned}
$$

where, $\mathrm{a}$ is the $\mathrm{X}$ coordinate of the LSC center, $\mathrm{b}$ is the $\mathrm{Y}$ coordinate of the LSC center and $\mathrm{n}$ is the number of coordinates of the profile taken for evaluation. The center $(a, b)$ calculated for the data points given by Wen et al. (1999) using X coordinate of the LSC center (8) and Y coordinate of the LSC center (9) is $(0.0726,-0.1060)$ from $(0,0)$. With the above value of $(a, b)$ nine other centers were chosen randomly and is given in the Table 1 which are used as the initial population for the evaluation of roundness 
error using TLBO algorithm. The code for TLBO algorithm was developed using MATLAB R2016a. The minimum roundness error and its corresponding centre coordinate obtained at the end of $100^{\text {th }}$ iteration is compared against the results of PSO and is given in the Table 2.

Table 1

Centre co-ordinates of least square circle from profile centre

\begin{tabular}{ccc}
\hline S. & a & Centre coordinate \\
No. & 0.0726 & b \\
\hline 1 & 0.0714 & -0.1060 \\
2 & 0.0698 & -0.1051 \\
3 & 0.0730 & -0.0999 \\
4 & 0.0699 & -0.1062 \\
5 & 0.0726 & -0.1060 \\
6 & 0.0731 & -0.1069 \\
7 & 0.0721 & -0.1042 \\
8 & 0.0692 & -0.1055 \\
9 & 0.0690 & -0.1056 \\
10 & & -0.1060 \\
\hline
\end{tabular}

Fig. 3 shows the evaluation of roundness error for 100 iterations using TLBO algorithm based on MZC method.

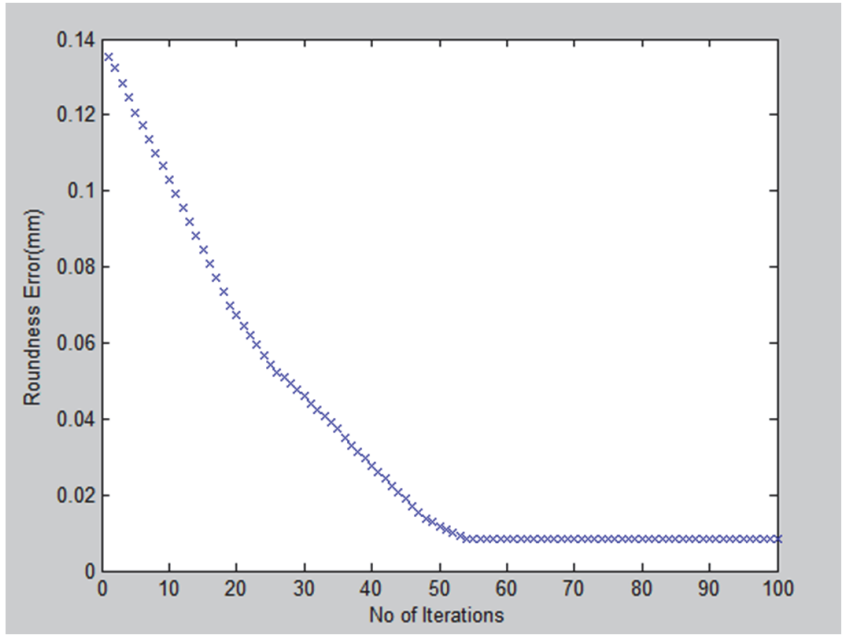

Fig. 3. Evaluation of roundness error for 100 iterations using TLBO algorithm based on MZC method

Table 2

Centre co-ordinates of MZC obtained using TLBO and PSO algorithms

\begin{tabular}{cccc}
\hline $\begin{array}{c}\text { S. } \\
\text { No }\end{array}$ & Method & Centre coordinates $(\mathbf{m m})$ & Roundness error $(\mathbf{m m})$ \\
\hline 1 & TLBO & $\begin{array}{c}(0.035625187904716, \\
-0.052938509298430)\end{array}$ & 0.008560071580591 \\
2 & PSO & $\begin{array}{l}(0.035614971220104, \\
-0.052929481200396)\end{array}$ & 0.008537464354626 \\
\hline
\end{tabular}

\section{Result and discussion}

The results of TLBO algorithm are compared with PSO algorithm on the following aspects. 


\subsection{Number of iterations Vs Roundness error}

The MZC roundness error obtained using TLBO and PSO algorithms for different number of iterations, the percentage variation between the results and time taken by the two algorithms to converge to the minimum roundness error are given in the Table 3 . The minimum zone circles obtained using TLBO algorithm for the data points using MATLAB R2016a is shown in Fig. 4.

Table 3

Roundness error, \% variation and time taken for various numbers of iterations

\begin{tabular}{|c|c|c|c|c|c|c|}
\hline \multirow[t]{2}{*}{ S. No } & \multirow[t]{2}{*}{$\begin{array}{l}\text { Number of itera- } \\
\text { tions }\end{array}$} & \multicolumn{2}{|c|}{ Roundness error (in mm) } & \multirow{2}{*}{$\begin{array}{l}\text { Variation be- } \\
\text { tween the results } \\
\text { of roundness } \\
\text { error } \\
\text { (in \%) }\end{array}$} & \multicolumn{2}{|c|}{$\begin{array}{l}\text { Time taken for con- } \\
\text { vergence } \\
\text { (in sec) }\end{array}$} \\
\hline & & TLBO & PSO & & TLBO & PSO \\
\hline 1 & 10 & 0.085984631954501 & 0.062906202262788 & 26.84 & 1.31 & 0.25 \\
\hline 2 & 50 & 0.008923032338120 & 0.008537470731353 & 4.32 & 4.69 & 0.91 \\
\hline 3 & 100 & 0.008560071580591 & 0.008537464354626 & 0.264 & 7.65 & 1.80 \\
\hline 4 & 150 & 0.008542355751479 & 0.008537464354593 & 0.057 & 10.97 & 2.40 \\
\hline
\end{tabular}

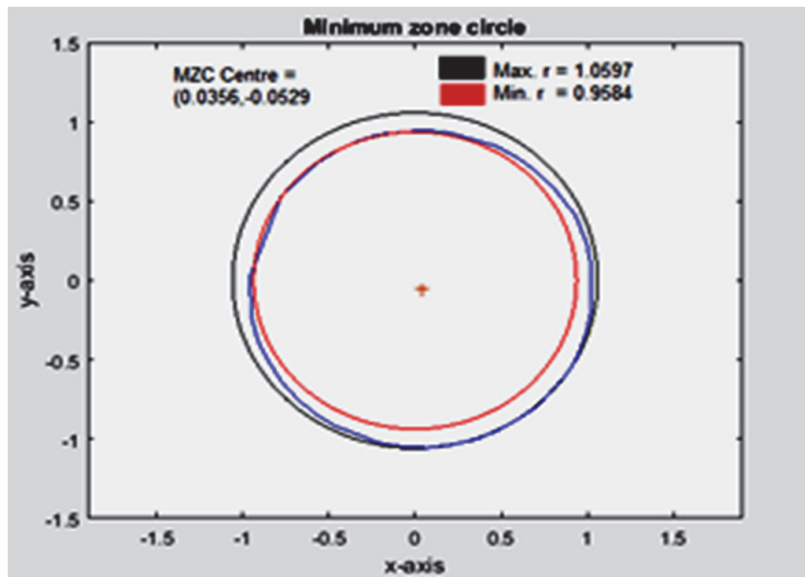

Fig. 4. Minimum zone circles obtained using TLBO

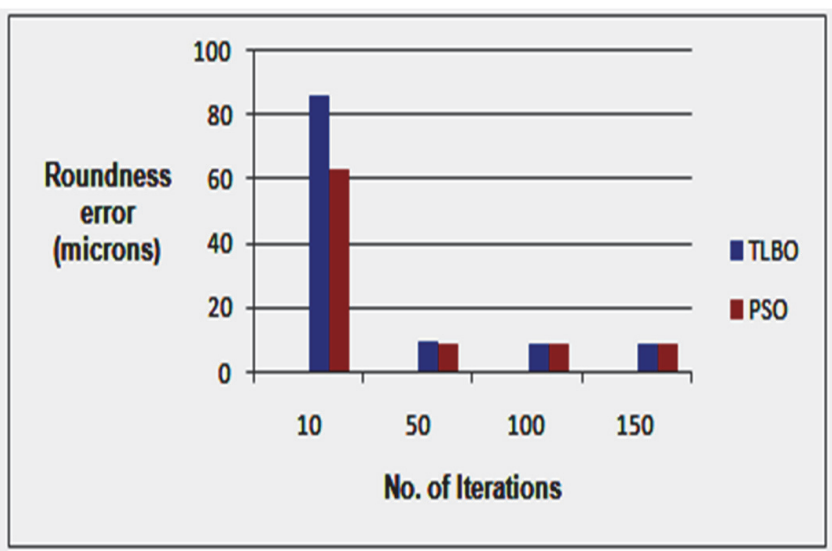

Fig. 5. Comparison of roundness error in terms of number of iterations

It is observed that the roundness error obtained using TLBO algorithm decreases with increase in number of iterations and becomes stable after 100 iterations. It is also seen that both algorithms provide the roundness error same as obtained by Du et al. (2014) and Wen et al. (1999). Also, the algorithm specific parameter used in PSO such as inertia weight required a lot of trials to find the optimum values to be used for the evaluation of roundness error. But TLBO algorithm does not require any specific parameters to be tuned which becomes an advantage. The only drawback with the TLBO algorithm is the increased convergence time than the PSO algorithm. Nevertheless, the roundness error provided by both the algorithms is same. The percentage difference between the results provided by TLBO and PSO is only 0.264 for 100 iterations and decreases even further when the number of iterations is increased. Comparison of roundness error obtained using both the algorithms for the co-ordinate points presented in Jywe et al. (1999) in terms of number of iterations is shown in Fig. 5.

\section{Conclusion}

Thus the TLBO algorithm is introduced for form measurement and found to be effective for the evaluation of roundness error. The codes were developed for both the algorithms and the efficiency of TLBO algorithm was checked in terms of number of iterations and computation time. It was found that both 
TLBO and PSO algorithms provide same result after 100 iterations but the time taken for TLBO algorithm to converge to minimum roundness error was more than PSO algorithm. Any optimization algorithm requires specific parameters to be tuned to obtain the optimum value. It is difficult to set these parameters as each application requires different parameters to be tuned. But TLBO does not require tuning of any algorithm-specific parameters which becomes the major advantage of TLBO algorithm.

\section{Acknowledgement}

The authors thank PSG College of Technology, Peelamedu Coimbatore, India, for providing necessary infrastructure and facilities to complete this work.

\section{References}

Du, C. L., Luo, C. X., Han, Z. T., \& Zhu, Y. S. (2014). Applying particle swarm optimization algorithm to roundness error evaluation based on minimum zone circle. Measurement, 52, 12-21.

Dhanish, P. B. (2002). A simple algorithm for evaluation of minimum zone circularity error from coordinate data. International Journal of Machine Tools and Manufacture, 42(14), 1589-1594.

Farooqui, S. A., Doiron, T., \& Sahay, C. (2009). Uncertainty analysis of cylindricity measurements using bootstrap method. Measurement, 42(4), 524-531.

He, G., Zhang, M., \& Song, Z. (2015). Error evaluation of free-form surface based on distance function of measured point to surface. Computer-Aided Design, 65, 11-17.

Jywe, W. Y., \& Liu, C. H. (1999). The min-max problem for evaluating the form error of a circle. Measurement, 26(4), 273-282.

Lei, X., Zhang, C., Xue, Y., \& Li, J. (2011). Roundness error evaluation algorithm based on polar coordinate transform. Measurement, 44(2), 345-350.

Mekid, S., \& Vacharanukul, K. (2011). In-process out-of-roundness measurement probe for turned workpieces. Measurement, 44(4), 762-766.

Muralikrishnan, B., \& Raja, J., (2009). Computational Surface and Roundness Metrology. Springer $\left(1^{\text {st }}\right.$ ed). London: Springer-Verlag.

Rao, R., \& Patel, V. (2013). Comparative performance of an elitist teaching-learning-based optimization algorithm for solving unconstrained optimization problems. International Journal of Industrial Engineering Computations, 4(1), 29-5

Rao, R. V. (2015). Teaching learning based optimization algorithm: and its engineering applications. Springer.

Sun, T. H. (2009). Applying particle swarm optimization algorithm to roundness measurement. Expert Systems with Applications, 36(2), 3428-3438.

Wen, X., Xia, Q., \& Zhao, Y. (2006). An effective genetic algorithm for circularity error unified evaluation. International Journal of Machine Tools and Manufacture, 46(14), 1770-1777.

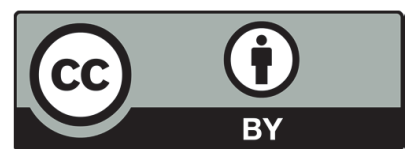

(C) 2018 by the authors; licensee Growing Science, Canada. This is an open access article distributed under the terms and conditions of the Creative Commons Attribution (CCBY) license (http://creativecommons.org/licenses/by/4.0/). 